\title{
RENORMALIZATION GROUP THEORY OF THE CRITICAL PROPERTIES OF THE INTERACTING BOSE FLUID
}

\author{
Richard J. CRESWICK and Frederik W. WIEGEL \\ Department of Applied Physics, Twente University of Technology, 7500 AE Enschede, The Netherlands
}

Received 10 June 1982

\begin{abstract}
Starting from a functional integral representation of the partition function we apply the renormalization group to the interacting Bose fluid. A closed form for the renormalization equation is derived and the critical exponents are calculated in $4-\epsilon$ dimensions.
\end{abstract}

In view of the ongoing development of the renormalization group the application of this technique to quantum fluids continues to be of considerable interest. This problem has been studied by several authors [113] within different approximations. In this note we develop an approximation which leads to a closed form expression for the infinitesimal renormalization transformation. The advantage of such a closed form expression is that: (a) it permits one to study the global properties of the renormalization group for quantum fluids; (b) it enables one to interpret the transformation as a nonlinear dynamics, which raises the possibility of applying concepts from this field.

The grand canonical partition function, $Z(\beta, \mu)$, for the interacting Bose gas may be represented as a functional integral over the complex scalar fields $\phi(x$, $\theta)[14,15]$

$Z(\beta, \mu)=\int D[\phi] \mathrm{e}^{-S_{0}[\phi]-U[\phi]}$,

where

$S_{0}[\phi]=\int \mathrm{d}^{D} \boldsymbol{x} \int_{0}^{1} \mathrm{~d} \theta\left(\frac{1}{\beta} \phi^{*} \frac{\partial \phi}{\partial \theta}+\frac{1}{2}|\nabla \phi|^{2}\right)$,

and

$U[\phi]=\int \mathrm{d}^{D} \boldsymbol{x} \int_{0}^{1} \mathrm{~d} \theta\left(-\mu|\phi|^{2}+\frac{1}{2} g|\phi|^{4}\right)$.
In these formulae we use units such that $\hbar=1$, the boson mass $=1$ and the cutoff in $k$ space $\Lambda=1$. Occasionally we shall use the symbol $\phi(x)$ for $\phi(x, \theta)$. For derivations and details of the notation we refer to ref. [9]. Following this same reference we implement the renormalization transformation of Wilson in two steps. First the field $\phi$ is separated into a component $\phi^{(\mathrm{L})}$ with long wavelengths $\left.0 \leqslant|k| \leqslant 1-\eta\right)$ and a component $\phi(S)$ with short wavelengths $(1-\eta$ $\leqslant|k| \leqslant 1$ ). The partial functional integration is performed over the fields $\phi^{(S)}$, and $\phi^{(L)}$ is rescaled in order to restore the cutoff to unity. We shall always work to $\mathrm{O}(\eta)$, and ultimately consider the limit $\eta \rightarrow 0$.

The partial functional integration may be carried out analytically with the approximation that the free Green function $G^{(\mathbf{S})}$ of the fields $\phi^{(\mathrm{S})}$,

$G^{(\mathrm{S})}\left(x, x^{\prime}\right)=\left\langle\phi^{(\mathrm{S})}(x) \phi^{*}(\mathrm{~S})\left(x^{\prime}\right)\right\rangle$,

varies rapidly compared to a function $f\left[\phi^{(\mathrm{L})}(x)\right]$ of $\phi^{(\mathrm{L})}$, so that we may write

$f\left[\phi^{(\mathrm{L})}(x)\right] G^{(\mathrm{S})}\left(x, x^{\prime}\right) \approx G^{(\mathrm{S})}\left(x, x^{\prime}\right) f\left[\phi^{(\mathrm{L})}\left(x^{\prime}\right)\right]$.

The second step in the renormalization transformation consists of rescaling the variables:

$k=(1-\eta) k^{(\mathrm{R})}, \quad x=(1-\eta)^{-1} x^{(\mathrm{R})}$,

$\phi=(1-\eta)^{(D-2) / 2} \phi^{(\mathrm{R})}, \quad \beta=(1-\eta)^{-2} \beta^{(\mathrm{R})}$.

This restores the momentum cutoff to unity and we are left with a functional integral of the same form. 
The renormalized interaction is given to first order in $\eta$ by

$$
\begin{aligned}
& U^{(\mathrm{R})}=U+\eta\left[D U-(D-2)|\phi|^{2} U^{\prime}+\left[\sigma_{D} /(2 \pi)^{D}\right]\right. \\
& \left.\times \ln \left(\frac{\sinh \frac{1}{4} \beta\left[\left(1+2 U^{\prime}\right)\left(1+2 U^{\prime}+4|\phi|^{2} U^{\prime \prime}\right)\right]^{1 / 2}}{\sinh \frac{1}{4} \beta\left[1+2 U^{\prime}(\phi=0)\right]}\right)\right] .
\end{aligned}
$$

In (6) the prime indicates differentiation with respect to $|\phi|^{2}$ and $\sigma_{D}$ is the area of a $D$-sphere. We have specialized to potentials which are local functions of $|\phi|^{2}$ only. Details of the derivation will appear in a more expanded paper [16]. One of the nice features of this method is that the local gauge invariance of the potential is preserved under renormalization. In (6) we have subtracted the $\phi$-independent part from $U^{(\mathrm{R})}$, so that $U^{(\mathrm{R})}(\phi=0) \equiv 0$. These terms are taken out of the functional integral and contribute to the thermodynamic potential.

We now define a parameter $t_{n}=(1-\eta)^{n}$ and take the limit $\eta \rightarrow 0$. In this limit $t_{n}$ becomes a continuous parameter $t$ and we find for our renormalization equation

$$
\begin{aligned}
& -t \partial U / \partial t=D U-(D-2)|\phi|^{2} U^{\prime}+\left[\sigma_{D} /(2 \pi)^{D}\right] \\
& \times \ln \left(\frac{\sinh \frac{1}{4} \beta t^{2}\left[\left(1+2 U^{\prime}\right)\left(1+2 U^{\prime}+4|\phi|^{2} U^{\prime \prime}\right)\right]^{1 / 2}}{\sinh \frac{1}{4} \beta t^{2}\left[1+2 U^{\prime}(\phi=0, t)\right]}\right) .
\end{aligned}
$$

The sum of the "constant terms" mentioned above becomes an integral over $t$, and yields a part of the canonical pressure

$$
\begin{aligned}
p^{\prime} & =-\frac{1}{\beta} \frac{\sigma_{D}}{(2 \pi)^{D}} \int_{0}^{1} \mathrm{~d} t t^{D-1} \\
& \times \ln \left(\frac{\sinh \frac{1}{4} \beta t^{2}\left[1+2 U^{\prime}(\phi=0, t)\right]}{\sinh \frac{1}{4} \beta t^{2}}\right) .
\end{aligned}
$$

In the case of Bose condensation one must relax the condition that the potential is a function of $|\phi|^{2}$ only. Following the method of Bogoliubov [17] we write

$$
U_{\mathrm{BC}}[\phi]=U\left[\left|\phi_{0}+\phi\right|^{2}\right]-U\left[\left|\phi_{0}\right|^{2}\right] \text {. }
$$

The new parameter $\phi_{0}$ is the "condensate wave function", and is a function of $t$ only. Substituting $U_{\mathrm{BC}}$ into the renormalization equation we find that $\phi_{0}$ has the simple scaling behavior $\phi_{0}(t)=\sqrt{n_{0}} t^{-(D-2) / 2}$.

The equilibrium value of $n_{0}$, the condensate density, is found by maximizing the canonical pressure [18]

$\partial p\left(\beta, \mu, n_{0}\right) / \partial n_{0}=0$.

After many renormalizations, in the limit $t \rightarrow 0$, the renormalization equations have the slightly simpler form

$$
\begin{aligned}
& -t \partial f / \partial t=D f^{\prime}-(D-2) x f^{\prime} \\
& \quad+\frac{1}{2} \ln \left(\frac{\left(1+2 f^{\prime}\right)\left(1+2 f^{\prime}+4 x f^{\prime \prime}\right)}{\left[1+2 f^{\prime}(0, t)\right]^{2}}\right),
\end{aligned}
$$

where $x=\left[\sigma_{D} /(2 \pi)^{D}\right]^{-1}|\phi|^{2}$ and $U=\left[\sigma_{D} /(2 \pi)^{D}\right] f(x)$. The form of the infinitesimal renormalization transformation (12) is similar to the expression found by Nicoll et al. $[19,20]$ for classical systems. For $D$ $=4-\epsilon, f$ is of order $\epsilon$, and we may expand the logarithm in (12). One can show that $f$ has the form

$f=r(t) x+\frac{1}{2} s(t) x^{2}+\mathrm{O}\left(\epsilon^{3}\right)$.

Substitution of (13) into the renormalization equation yields to $O\left(\epsilon^{2}\right)$ for $r$ and $s$,

$-t \partial r / \partial t=2(1-4 s) r+4 s, \quad-t \partial s / \partial t=\epsilon s-20 s^{2}$.

These equations have the fixed point $r^{*}=-\epsilon / 10, s^{*}$ $=\epsilon / 20$, and near the fixed point

$r(t)=r^{*}+\left(r_{0}-r^{*}\right) t^{-2(1-\epsilon / 5),}$

$s(t)=s^{*}+\left(s_{0}-s^{*}\right) t^{\epsilon}$.

The behavior of $r(t)$ is responsible for the nonanalytic behavior of the pressure in (8). This gives for the critical exponent for the correlation length [9]

$\nu=\frac{1}{2}+\frac{1}{10} \epsilon$,

and for the specific heat

$\alpha=\frac{1}{10} \epsilon$.

We see from (10) that $n_{0}$ is also a relevant variable, and by (11) the critical exponent for the order parameter is

$\beta=\frac{1}{2}-\frac{3}{20} \epsilon$.

Assuming the scaling law $2 \beta-\eta \nu=(D-2) \nu$ we find that $\eta=0$. By the Josephson relation [21] the critical 
exponent for the superfluid density, $\sigma$, is given by $2 \beta-\eta \nu$, and for $\epsilon=1$ we find $\sigma=0.7$.

Experimentally for liquid ${ }^{4} \mathrm{He}, \alpha=0$ and $\sigma=0.67$ which suggests that our simple theory is not too unrealistic. Perhaps what is more surprising is that, to first order in $\epsilon$, the critical exponents of this quantum system are given by the classical $\epsilon$-expansion for a twocomponent order parameter. Several authors [1-13] have observed that this is a consequence of the scaling of the "quantum parameter" $\beta \hbar^{2} / m$, which under successive renormalizations tends to zero. We are led to the conclusion that if the system at the critical line is described by a fixed point, then there exists a classical system with the same critical behavior, though not necessarily the same phase diagram.

This work is part of the research program of the "Stichting voor Fundamenteel Onderzoek der Materie" (FOM), which is financially supported by the "Nederlandse Organisatie voor Zuiver Wetenschappelijk Onderzoek" (ZWO).

\section{References}

[1] K.K. Singh, Phys. Lett. 51A (1975) 27.

[2] K.K. Singh, Phys. Rev. B12 (1975) 2819.
[3] K.K. Singh, Phys. Lett. 57A (1976) 309.

[4] K.K. Singh, Phys. Rev. B13 (1976) 3192.

[5] A.D. Stella and F. Toigo, Nuovo Cimento 34B (1976) 207.

[6] M. Baldo, E. Catara and U. Lombardo, Lett. Nuovo Cimento 15 (1976) 214.

[7] L. De Cesare, Lett. Nuovo Cimento 22 (1978) 325.

[8] F.W. Wiegel, Physica 91A (1978) 139.

[9] F.W. Wiegel, in: Path integrals, eds. G.J. Papadopoulos and J.T. Devreese (Plenum, New York, 1978) p. 419.

[10] J.C. Lee, Phys. Rev. B20 (1979) 1277.

[11] J.C. Lee, Physica 104A (1980) 189.

[12] G. Busiello and L. De Cesare, Phys. Lett. 77A (1980) 177.

[13] G. Busiello and L. De Cesare, Nuovo Cimento 59B (1980) 327.

[14] R.J. Creswick, Ph.D. Thesis, Department of Physics, University of California, Berkeley (1981).

[15] F.W. Wiegel, Phys. Rep. 16 (1975) 57.

[16] R.J. Creswick and F.W. Wiegel, to be published.

[17] N.N. Bogoliubov, J. Phys. USSR 11 (1947) 23.

[18] A.L. Fetter and J.D. Walecka, Quantum theory of many particle systems (McGraw-Hill, New York, 1971) p. 206.

[19] J.F. Nicoll, T.S. Chang and H.E. Stanley, Phys. Rev. B12 (1975) 458.

[20] J.F. Nicoll, T.S. Chang and H.E. Stanley, Phys. Rev. A13 (1976) 1251.

[21] B.D. Josephson, Phys. Lett. 21 (1966) 608. 